\title{
Optimal Locations of Electric Public Charging Stations Using Real World Vehicle Travel Patterns
}

\author{
Narges Shahraki ${ }^{1,2}$, Hua Cai ${ }^{2,3}$, Metin Turkay ${ }^{1}$ and Ming $\mathrm{Xu}^{2,3, *}$ \\ ${ }^{1}$ Department of Industrial Engineering, Koç University, Istanbul 34450, Turkey \\ ${ }^{2}$ School of Natural Resources and Environment, University of Michigan, Ann Arbor, MI 48109-1041, United States \\ ${ }^{3}$ Department of Civil and Environmental Engineering, University of Michigan, Ann Arbor, MI 48109-2125, United States
}

\begin{abstract}
We propose an optimization model based on vehicle travel patterns to capture public charging demand and select the locations of public charging stations to maximize the amount of vehicle-miles-traveled (VMT) being electrified. The formulated model is applied to Beijing, China as a case study using vehicle trajectory data of 11,880 taxis over a period of three weeks. The mathematical problem is formulated in GAMS modeling environment and Cplex optimizer is used to find the optimal solutions. Formulating mathematical model properly, input data transformation, and Cplex option adjustment are considered for accommodating large-scale data. We show that, comparing to the 40 existing public charging stations, the 40 optimal ones selected by the model can increase electrified fleet VMT by 59\% and $88 \%$ for slow and fast charging, respectively. Charging demand for the taxi fleet concentrates in the inner city. When the total number of charging stations increase, the locations of the optimal stations expand outward from the inner city. While more charging stations increase the electrified fleet VMT, the marginal gain diminishes quickly regardless of charging speed.
\end{abstract}

Keywords: Optimization, electric vehicles, vehicle trajectory, charging infrastructure planning

\section{Introduction}

Fossil fuel-based road transportation has instigated increasing global demand for oil and air pollution, especially in urban areas (Hoogma et al., 2005). Emerging vehicle technologies that can utilize alternative fuels are considered as potential solutions for these issues, such as electric vehicles (EVs). Although the life cycle environmental implications of EVs depends on the fuel mix of electricity generation (Torchio and Santarelli, 2010), using electricity instead of liquid fossil-based fuels for road transportation can relocate tailpipe emissions from mobile vehicular sources to stack emissions in power plants which are more concentrated and easier to control. Many countries have set goals for EV adoption. For example, the U.S. plans to have more than 1.8 million plug-in hybrid electric vehicles (PHEVs); and China hopes to put 5 million hybrid and electric vehicles on the road by 2020 (Deutsche Bank Group, 2012; Navigant Research Group, 2013).

\footnotetext{
${ }^{*}$ Corresponding author at: 440 Church Street, Ann Arbor, MI 48109-1041, United States. Tel: +1 7347638644 ; fax: +1 734936 2195. E-mail address: mingxu@umich.edu (M. Xu).
} 
One of the factors that significantly impact the growth of the EV market is access to public charging infrastructure (Morrow, 2008). Many governments are investing in the deployment of public charging stations. For example, California has announced to build 200 public fastcharging stations; and British Columbia, Canada has set goals for building 570 charging stations across the province (City of Surrey, 2012; JR, 2012). While the deployment of charging infrastructure has been moving forward in many cities, research on developing mathematical models for charging infrastructure siting is also growing. Xi et al., (2013) have formulated an optimization framework for charging station siting to maximize the amount of energy and the expected EVs recharged, by estimating charging demand as a function of household demographic variables (e.g. average total mileage driven) and macroeconomic variables (e.g. gasoline and electricity prices). Sathaye and Kelley (2013) estimate charging demand using a linear function of two terms - traffic and population - to minimize total distances between demand locations and charging stations. Sadeghi-Barzani et al., (2014) predict the charging demand based on the number of EV owners to minimize the total cost associated with charging which includes travel cost to stations and electricity cost. He et al., (2013) predict charging demand based on travel time, charging expenses, availability, and attractions to maximize total social welfare. Dong et al., (2014) use multiday travel data of 275 household in the Seattle metropolitan area to minimize the number of trips which cannot be completed using electricity. Despite the difference on scope and goals, these studies use non-exact algorithms to find local optimal solutions to our knowledge.

Two research gaps exist in the literature on siting public charging stations. First, methods currently used in estimating charging demand may not reflect the real world situation. Unlike refueling liquid fuels which only takes a few minutes to fill the tank, fully recharging the battery on an EV can take a much longer time, from 30 minutes to several hours depending on the charger power, battery size, and the state of charge of the battery (Dong et al., 2014). Therefore, EV charging is more likely to happen at the end of a trip instead of in the middle of a trip. In addition, EV owners can charge their vehicles at home during the night. As a result, using traffic flow volume or vehicle ownership density to estimate charging demand as predominately used in previous studies may not be valid. Secondly, few studies considered environmental impacts of EV charging as the objective function for global optimal solution. The ultimate goal of EV system deployment is to fulfill more travel needs using electricity instead of fossil-based liquid fuels. We aim to address both gaps in this study by 1) using large-scale real world vehicle travel data to better model charging demand and 2) maximizing electrified fleet vehicle-miles-traveled (VMT) as the objective function to find the global optimal solution. Our previous work has demonstrated that collective public parking "hotspots" extracted from real world vehicle trajectory data are good indicators of public charging demand (Cai et al., 2014). This research expands upon Cai et al., (2014) to develop an optimization model to solve for global optimization solutions for public charging station siting using large-scale real world vehicle travel data. In addition, the goal of our optimization model is to maximize electrified fleet VMT, which directly links to the environmental benefits of vehicle electrification. 
Using Beijing, China as a case study, this paper presents an optimization framework which utilizes large-scale real world vehicle trajectory data for selecting the location of public charging stations to maximize electrified fleet VMT. The demonstrated optimization framework can be applied to other fleets in other cities using similar data. The case study also has its own policy relevance because Beijing plans to deploy 200,000 electric vehicles on road by 2017 and build 10,000 public charging stations (NAATBatt International, 2015; WantChinaTimes, 2013). Results from this study can help future decisions on developing public charging stations in Beijing. The proposed optimization model is implemented in GAMS with Cplex solver. In order to accommodate for the large-scale vehicle trajectory data in the model, we identify factors in model formulation, input data format, and settings of Cplex options that need to be adjusted to solve the model efficiently. In summary, major contributions of this paper include 1) formulating an optimization model which selects the location of public charging stations to maximize electrified fleet VMT; 2) incorporating vehicle travel patterns by using large-scale individual-based vehicle trajectory data to model public charging demand; 3) studying public charging infrastructure planning in Beijing as a case study; and 4) providing suggestions in model formulation and execution to handle largescale data.

\section{Mathematical formulation}

EVs include battery electric vehicles (BEV) and plug-in hybrid electric vehicles (PHEV). BEVs use electricity as the sole power source while PHEV has the flexibility of using both electricity and liquid fuels (Markel, 2010). In this paper we focus on PHEVs to allow drivers finishing trips on liquid fuels when batteries are depleted, because it is unclear how limited driving range of BEVs will affect the behavior of drivers.

Let $G(i, j, k)$ be a network with $i$ candidate locations for installing public charging stations, $j$ individual PHEVs, and $k$ trips for each vehicle in the examined period. The time spent between two consecutive trips is defined as the dwell time.

Each vehicle $(j)$ has a remained battery charge $\left(R_{j k}\right)$ at the end of each trip $(k)$ before starting its dwell time. For the convenience of modeling, $R_{j k}$ is measured as the mileage that the vehicle can travel with the remaining electricity (battery range). $R_{j k}$ can be formulated as shown in Eq. (1) (Dong et al., 2014). Negative values of $R_{j k}$ represent the mileage that cannot be powered by electricity (i.e., powered by liquid fuels) in trip $k$. Eq. (2) shows the real remaining battery range $\left(\hat{R}_{j k}\right)$ of vehicle $j$ at the end of trip $k$, which is forced to be nonnegative.

$$
\begin{aligned}
& R_{j k}=\hat{R}_{j k-1}+E_{j k-1}-d_{j k} \quad \forall j \in J, \forall k \in K \\
& \hat{R}_{j k-1}=\max \left\{R_{j k-1}, 0\right\} \quad \forall j \in J, \forall k \in K
\end{aligned}
$$

where $R_{j k}$ is the remaining battery range of vehicle $j$ at the end of trip $k$ (mile), $E_{j k-1}$ is the electricity recharged (measured in miles) for vehicle $j$ during the dwell time between trip $k-1$ and trip $k$; and $d_{j k}$ is the travel distance (miles) of vehicle $j$ during trip $k$. 
This model differentiates home charging and public charging. If vehicle $j$ does not park at home after trip $k\left(h_{j k}=0\right)$, the vehicle seeks public charging opportunities. Electricity recharged for vehicle $j$ after trip $k\left(E_{j k}\right)$, as shown in Eq. (3), equals to the difference between the full battery range and the remaining battery range if the dwell time is longer than what is required to fully charge the battery, or the exact amount of electricity that can be charged if a full charge cannot be achieved within the dwell time. $E_{j k}$ equals to 0 if no charging station is available for vehicle $j$ at the end of trip $k$. If vehicle $j$ parks at home after trip $k\left(h_{j k}=1\right)$, home charging is utilized. Recharged electricity at home, as shown in Eq. (4), equals to the difference between the full battery range and the remaining battery charge if the home parking time is longer than what is required to fully charge the battery, or the exact amount of electricity that can be charged during home dwell time.

$$
\begin{array}{ll}
E_{j k}=\min \left\{E_{j}-\hat{R}_{j k}, \frac{L e t_{j k}}{r_{j}}, M \sum_{i \in I} P_{i j k}\right\} & \text { if } h_{j k}=0 \\
E_{j k}=\min \left\{E_{j}-\hat{R}_{j k}, \frac{L h t_{j k}}{r_{j}}\right\} & \text { if } h_{j k}=1
\end{array}
$$

where $h_{j k}$ equals to 1 if vehicle $j$ is parked at home after trip $k$ and 0 otherwise; $E_{j}$ is the effective all-electric-range (AER) of vehicle $j$ 's battery (measured in miles); Le is the charging power level $(\mathrm{kW})$ at each of the public charging stations (assuming same for all public charging stations); $L h$ is home charging power level; $t_{j k}$ is dwell time of vehicle $j$ at the end of trip $k$ (hour); $r_{j}$ is the average electricity consumption rate of vehicle $j$ in charge depletion (CD) mode $(\mathrm{kWh} / \mathrm{mile}) ; M$ is a large number greater than $E_{j}$; and $P_{i j k}$ is the availability of charging station for vehicle $j$ at location $i$ after trip $k . P_{i j k}$ equals to 1 if candidate location $i$ is accessible for vehicle $j$ at the end of trip $k$ and a charging station is installed at location $i$. Accessibility of charging station at location $i$ by vehicle $j$ is measured by the distance between location $i$ and vehicle $j$ at the end of trip $k$. If this distance is less than the service range of charging stations, location $i$ is accessible.

\subsection{Optimization model}

The optimal selection of charging station locations in a geographical area using the travel patterns of individual vehicles defined in this paper is given by Eqs. (5) - (14). The objective function, as shown in Eq. (5), minimizes the total travel distances that cannot be fulfilled by electricity. This is equivalent as maximizing the electrified fleet VMT. Eqs. (6), (7) and (8) formulate the remaining battery range of vehicle $j$ at the end of trip $k$. In Eq. (7), $R_{j}$ is the remaining battery range of vehicle $j$ at the beginning of trip 1 . Recharged electricity of vehicle $j$ at the end of trip $k$ is shown in Eqs. (9) and (10). Eq. (11) shows the budget constraint, limiting the maximum number of public charging stations as $B$. Charging opportunity is available at candidate location $i$ for vehicle $j$ at the end of trip $k$ if two conditions are satisfied simultaneously, as shown in Eq. (12). The first condition is that the distance between candidate location $i$ and the location of vehicle $j$ at the end of trip $k$ is less than the specified charging station service range. If vehicle $j$ at the end of trip $k$ is within the service range of candidate location $i, Z_{i j k}$ equals to 1 , otherwise zero. The second condition is that a charging 
station is installed at location $i\left(y_{i}=1\right)$. The model solves $y_{i}$ for the optimal solutions. Eq. (13) and (14) show the binary and positive variables, respectively.

$\min \sum_{k \in K} \sum_{j \in J}\left(\hat{R}_{j k}-R_{j k}\right)$

Subject to:

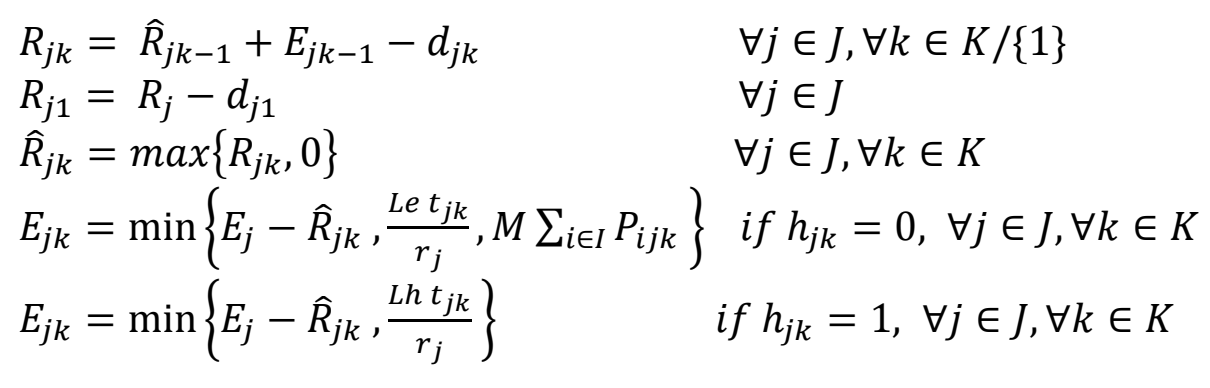

$\sum_{i \in I} y_{i} \leq B$

$P_{i j k}=Z_{i j k} y_{i}$

$\forall j \in J, \forall k \in K, \forall i \in I$

$P_{i j k}, y_{i} \in\{0,1\}$

$\forall j \in J, \forall k \in K, \forall i \in I$

$\hat{R}_{j k}, E_{j k} \geq 0$

$\forall j \in J, \forall k \in K$

where the decision variables of the problem are as follows:

$R_{j k}: \quad$ The remaining battery range of vehicle $j$ at the end of trip $k$ (mile)

$\hat{R}_{j k}$ : The real remaining battery range of vehicle $j$ at the end of trip $k$ (mile)

$P_{i j k}$ : Binary variable which shows the availability of public charging for vehicle $j$ at the end of trip $k$ at location $i$, with 1 indicating available and otherwise 0

$y_{i}$ : $\quad$ Binary variable which shows whether a charging station is installed at location $i$, with 1 indicating present and otherwise 0

$E_{j k}: \quad$ Battery electricity recharged for vehicle $j$ at the end of trip $k$ (mile)

The formulated optimization model is a Mixed Integer Non-Linear Programming (MINLP) model due to max and min operators in Eqs. (8), (9) and (10). Since solving MINLP problem is much more computationally challenging than solving a Mixed Integer Linear Programming (MILP) problem, we transform the MINLP to MILP in next section.

\subsection{Linearization}

By substituting Eq. (6) and (7) in Eq. (5), the objective function can be written in Eq. (15) which is equal to Eq. (16) for specific $j$. Therefore, the objective function can be written as in Eq. (17).

$\sum_{k \in K} \sum_{j \in J}\left(\hat{R}_{j k}-R_{j k}\right)=$

$\sum_{k=1} \sum_{j \in J}\left(\hat{R}_{j k}-E_{j}+d_{j k}\right)+\sum_{k>1 \& k \in K} \sum_{j \in J}\left(\hat{R}_{j k}-\hat{R}_{j k-1}-E_{j k-1}+d_{j k}\right)$

$\sum_{k \in K}\left(\hat{R}_{j k}-R_{j k}\right)=$

$\hat{R}_{j 1}-E_{j}+d_{j 1}+\hat{R}_{j 2}-\hat{R}_{j 1}-E_{j 1}+d_{j 2}+\hat{R}_{j 3}-\hat{R}_{j 2}-E_{j 2}+d_{j 3}+\cdots+\hat{R}_{j k}-\hat{R}_{j k-1}-$ 
$E_{j k-1}+d_{j k}=\hat{R}_{j k}-E_{j}+d_{j 1}+\sum_{k>1 \& k \in K}\left(-E_{j k-1}+d_{j k}\right)$

$\sum_{k \in K} \sum_{j \in J}\left(\hat{R}_{j k}-R_{j k}\right)=$

$\sum_{j \in J}\left(\hat{R}_{j k}-E_{j}+d_{j 1}+\sum_{k>1 \& k \in K}\left(-E_{j k-1}+d_{j k}\right)\right)$

According to Eqs. (17) and (5), we maximize the amount of recharged electricity $\left(E_{j k}\right)$. Therefore, Eqs. (18), (19), (20) and (21) show the linear formulation of Eqs. (9) and (10). To linearize Eq. (8), we add Eqs. (22) and (23) as constraints to the model and penalty function, Eq. (24), to the objective function with $\mu=1$. In addition, we multiply Eq. (5) by a coefficient $(M)$ which is a large number greater than $\mu$. Therefore, the objective function is changed to Eq. (25).

Adding the penalty function with $\mu=1$, Eq. (24), does not affect the optimal solution, since the value of $\left(\hat{R}_{j k}-R_{j k}\right)$ depends directly on the amount of recharged electricity $\left(E_{j k}\right)$ which objective function aims to maximize. Eqs. (6), (7) and (8) calculates the remaining battery range $\left(R_{j k}\right)$ at the end of each trip based on the amount of recharge electricity $\left(E_{j k}\right)$. The penalty function adjusts the value of $\hat{R}_{j k}$ to its minimum value which is $R_{j k}$. When $R_{j k}$ is negative, $\hat{R}_{j k}$ is set to zero, leading to that the penalty function will be zero. When $R_{j k}$ is positive, $\hat{R}_{j k}$ is set to its minimum value which is $R_{j k}$ according to Eq. (22). In addition, the large number $M$ guarantees that adding the penalty function does not have any effect on the optimal solution.

$$
\begin{array}{lr}
E_{j k} \leq E_{j}-\hat{R}_{j k} & \forall j \in J, \forall k \in K \\
E_{j k} \leq \frac{L e t_{j k}}{r_{j}} & \text { if } h_{j k}=0, \forall j \in J, \forall k \in K \\
E_{j k} \leq M \sum_{i \in I} P_{i j k} & \text { if } h_{j k}=0, \forall j \in J, \forall k \in K \\
E_{j k} \leq \frac{L h t_{j k}}{r_{j}} & \text { if } h_{j k}=1, \forall j \in J, \forall k \in K \\
\hat{R}_{j k} \geq R_{j k} & \forall j \in J, \forall k \in K \\
\hat{R}_{j k} \geq 0 & \forall j \in J, \forall k \in K \\
\mu \sum_{k \in K} \sum_{j \in J} \hat{R}_{j k} & \\
\min M \sum_{k \in K} \sum_{j \in J}\left(\hat{R}_{j k}-R_{j k}\right)+\mu \sum_{k \in K} \sum_{j \in J} \hat{R}_{j k}
\end{array}
$$

\subsection{Extensions on the optimization model}

In the case that a mix of charging stations with different charging power (e.g., fast charging and slow charging) need to be installed, the model can be extended by replacing Eqs. (9), (11) and (12) with Eqs. (26)-(33). Eq. (26) calculates the amount of electricity that vehicle $j$ can gain from recharge at either fast or slow charging stations at the end of trip $k$. Eqs. (27) and (28) show the limitation on the number of fast and slow charging stations, respectively. The amount of electricity that vehicle $j$ can get from a slow or fast charging station at the end of trip $k$ is formulated in Eqs. (29) and (30), respectively. Eq. (31) shows that at each candidate location, we can either install a fast charging station or a slow charging station, but not both. 
Eqs. (32) and (33) assure that each vehicle can only charge at one station at the end of each trip, with $l e^{s}$ and $l e^{f}$ representing the power level at a slow and fast charging stations, respectively.

$$
\begin{aligned}
& E_{j k}=\min \left\{E_{j}-\hat{R}_{j k}, \sum_{i \in I}\left(E_{j k i}^{S}+E_{j k i}^{f}\right)\right\} \quad \text { if } h_{j k}=0, \quad \forall j \in J, \forall k \in K \\
& \sum_{i \in I} y_{i}^{f} \leq B_{f} \\
& \sum_{i \in I} y_{i}^{S} \leq B_{S} \\
& E_{j k i}^{s}=\min \left\{\frac{l e^{s} t_{j k}}{r_{j}}, M Z_{i j k} y_{i}^{s}\right\} \quad \text { if } h_{j k}=0, \quad \forall j \in J, \forall k \in K, \forall i \in I \\
& E_{j k i}^{f}=\min \left\{\frac{l e^{f} t_{j k}}{r_{j}}, M Z_{i j k} y_{i}^{f}\right\} \quad \text { if } h_{j k}=0, \quad \forall j \in J, \forall k \in K, \forall i \in I \\
& y_{i}^{f}+y_{i}^{s} \leq 1 \\
& \forall i \in I \\
& \sum_{i \in I} E_{j k i}^{S} \leq \frac{l e^{s} t_{j k}}{r_{j}} \\
& \forall j \in J, \forall k \in K \\
& \sum_{i \in I} E_{j k i}^{f} \leq \frac{l e^{f} t_{j k}}{r_{j}} \\
& \forall j \in J, \forall k \in K
\end{aligned}
$$

In this formulation, the binary variable $y_{i}$ is replaced by the two binary variable sets to separate slow and fast charging stations. Similarly, $E_{i j k}$ is replaced by $E_{j k i}^{S}$ and $E_{j k i}^{f}$.

$y_{i}^{f}: \quad$ Binary variable which shows whether a fast charging station is installed at location

$i$, with 1 indicating presence and 0 indicating absence.

$y_{i}^{s}$ : $\quad$ Binary variable which shows if a slow charging station is installed at location $i$, with 1 indicating presence and 0 indicating absence.

$E_{j k i}^{S}$ : $\quad$ Electricity recharged for vehicle $j$ at the end of trip $k$ from a slow charging station at location $i$ (mile)

$E_{j k i}^{f}: \quad$ Electricity recharged for vehicle $j$ at the end of trip $k$ from a fast charging station $i$ (mile)

Constraints (26), (29) and (30) can be replaced by Eqs. (34)-(39) to obtain a linear formulation.

$$
\begin{array}{lrl}
E_{j k} \leq E_{j}-\hat{R}_{j k} & \forall j \in J, \forall k \in K \\
E_{j k} \leq \sum_{i \in I}\left(E_{j k i}^{S}+E_{j k i}^{f}\right) & \text { if } h_{j k}=0, \forall j \in J, \forall k \in K \\
E_{j k i}^{S} \leq \frac{l e^{s} t_{j k}}{r_{j}} & \text { if } h_{j k}=0, \forall j \in J, \forall k \in K, \forall i \in I \\
E_{j k i}^{S} \leq M Z_{i j k} y_{i}^{S} & \text { if } h_{j k}=0, \forall j \in J, \forall k \in K, \forall i \in I \\
E_{j k i}^{f} \leq \frac{l e^{f} t_{j k}}{r_{j}} & \text { if } h_{j k}=0, \forall j \in J, \forall k \in K, \forall i \in I
\end{array}
$$




\section{Data}

The taxi fleet in Beijing, China is used as a case study to apply the optimization model. Public fleets (i.e., taxis and buses) are likely early adopters for electric vehicles (A Krieger, L Wang, P Radtke, 2012). Although the case study is specific, the model can be generally applied to other fleets as well.

The vehicle trajectory data used in this study include 11,880 taxis (18\% of the fleet) in Beijing over a period of three weeks (March 2 to 25, 2009). The data were recorded by geographic positioning systems (GPS) devices and consist of vehicle id, time stamps up to the seconds, and position of the vehicle at the recorded time (longitude and latitude). A total of 255 million data points are included in the data, covering over 2 million trips and 34 million miles of travel. The trajectory data are used to evaluate vehicle travel behaviors and extract trip chains (a series of driving and parking events). A threshold of fifteen minutes parking is used to separate trips. This threshold is set with the consideration that drivers are not likely to go through the hassle of charging if they have less than 15 minutes of parking time. More information about this data set can be found in (Cai et al., 2014).

The existing 1,737 gas stations in Beijing are considered as candidate locations to build public charging stations. Wang et al., (2010) show that gas stations with charging capability may lead to more efficient long-term infrastructure use when EVs gradually replace conventional gasoline vehicles. Although it is unrealistic to expect drivers waiting for hours at gas stations for vehicle charging, taxi drivers normally do not stop for long duration during the day, making gas-station-based charging possible (Cai et al., 2014). Beijing currently has 40 charging stations built. To compare how charging station sites optimized with vehicle travel patterns can increase electrified fleet VMT, our baseline model selects 40 locations among the 1,737 candidates to build charging stations. The impacts of the total number of charging stations on electrified fleet VMT are also examined. The service range of each charging station is set at 1 mile with the assumption that drivers are not willing to significantly revise their travel behavior to accommodate for charging.

Vehicle travel behavior varies from day to day. To capture this variation and examine model sensitivity, we separate the trajectory data into three weekly data sets and apply model to each. For each week, the data are prepared into four input matrixes, $D\left(d_{j k}\right), T\left(t_{j k}\right), H\left(h_{j k}\right)$, and DIS (dis $i_{i j k}$ ), with $j$ equals to 11,881 , $i$ equals to 1,737 , and $k$ ranges from 83 to 125 depending on the week. Thus, each $d_{j k}, t_{j k}$ and $h_{j k}$ has at least 986,123 elements, where $d_{j k}$ is the travel distance of vehicle $j$ during trip $k$ (mile), $t_{j k}$ is dwell time of vehicle $j$ at the end of trip $k$ and before trip $k+1$ (hour), $h_{j k}$ is a $0-1$ matrix with 1 indicating that vehicle $j$ parks at home during its dwell time at the end of trip $k$ and 0 indicating that it does not park at home during the dwell time, and $d i s_{i j k}$ is the distance between candidate location $i$ and the location of vehicle $j$ at the end of trip $k$ (mile). Given the size of the data set, the DIS matrix has over one billion elements. If directly considering DIS matrix elements as inputs to our optimization model, we have to define a set of binary variables with over one billion elements and parameters to indicate the accessibility of DIS elements by each vehicle, which makes solving the problem 
computationally intractable. To make the problem solvable, the elements of the DIS matrix are translated into binary elements in a $Z$ matrix, where $Z_{i j k}$ is 1 if vehicle $j$ is parked within the service range of candidate location $i$ at the end of trip $k$, and zero otherwise. Using $Z$ matrix instead of DIS matrix reduces not only the data size but also the complexity of the problem by reducing the number of binary variables. However, reading $Z$ matrix with over one billion elements is still time-consuming. To overcome this computational obstacle, we use a specific feature from the optimization software GAMS. In GAMS if we do not set any value for a parameter, its default value is set to zero. Because the $Z$ matrix contains many zeros, we simply extract the non-zero $Z_{i j k}$ and store the $i, j$, and $k$ information in a series of vectors. Reading only the non-zero elements will reduce data size dramatically. In short, instead of directly using the DIS matrix, the information is translated into the position locations of nonzero $\mathrm{Z}_{i j k}$ as vectors before reading into the model.

We use $37.5 \mathrm{~kW}$ and $7.04 \mathrm{~kW}$ for power outputs of public fast charging and slow charging stations, respectively (State Gride Corporation of China, 2010; Tong, 2014). Home charging is assumed to use residential power outlet at $2.2 \mathrm{~kW}$. We assume all vehicles have access to home charging when they park at home. The effective all-electric-range $\left(E_{j}\right)$ of all PHEVs is assumed to be 100 miles. The impacts of this assumption on results is discussed in Section 4.3. Electricity consumption rate of PHEV is assumed to be constant at $0.35 \mathrm{kWh} / \mathrm{mile}$ during the charging-depleting mode (USA.gov, 2013). Variation of fuel economy due to different driving condition is not considered in this study. Charging efficiency is considered as $88 \%$ (Kelly et al., 2012). We assume that all vehicles start with fully charged battery at the beginning of each week, meaning that $R_{j}$ is set to 100 miles.

\section{Results and Discussions}

The optimization model is implemented in the GAMS environment using Cplex solver to solve the optimization problem. For solving MILP problems, Cplex uses a branch and cut algorithm which generates and solves a series of LP subproblems. The most common difficulty for solving MIP problems is running out of memory. Even small MILP problems generate many subproblems, making it very computationally intensive and requiring significant amount of physical memory. This problem arises when the branch and bound tree becomes so large that insufficient memory is available to solve an LP subproblem. The branch and bound tree can contain as large as $2^{n}$ terminal nodes, where $n$ equals the number of binary variables. A problem containing only 30 binary variables could produce a tree having over one billion terminal nodes (Solutions et al., 2012).

In our problem, we have 1,737 binary variables and three set of variables with $j \times k$ dimension, with $j$ equal to 11,881 and $k$ ranging from 83 to 125 . There are at least $3 \times 11,881 \times 83=$ $2,958,369$ general variables. When using GAMS/Cplex to find the global optimal solutions, the solution process using this dataset terminates with an unrecoverable integer failure message with 100 GB of physical memory that is highly demanding in terms of computing infrastructure. To overcome this problem, the values of following Cplex options are changed: reinv, varsel, cuts, nodesel, nodefileind, workmem, memoryemphasis, names, threads, and lpmethod. Justifying these options carefully can have dramatic effects on improving the 
computing speed and reducing memory usage. More details of these option adjustments are included in the Supplemental Information.

\subsection{The 40-station scenario}

At the time of this study, Beijing has 40 charging stations and posts already built. The 40station scenario implemented in our model finds the 40 optimal locations from the candidate gas stations based on weekly data. As shown in Figure 1a, while existing charging stations can electrify $29 \pm 3 \%$ and $35 \pm 2 \%$ of the fleet VMT with slow charging and fast charging respectively, location-optimized stations can effectively increase electrified fleet VMT to $46 \pm 4 \%$ and $66 \pm 2 \%$, on average an $59 \%$ and $88 \%$ improvement. Comparing to the locations of the existing stations, the optimized stations are concentrated in the inner city (Figure 1b). Here only the stations optimized with data for week 1 are presented for illustration. Location maps with other weekly data are included in the Supplemental Information (Figure SI-1). Similar patterns exist across all weeks. It is notable that, while the location of optimized stations in the suburban area varies from week to week, selection of optimized stations in the inner city is quite consistent (Figure SI-1d). By zooming into the inner city, it is clear that the locations of the optimized stations are quite different from those existing ones (Figure 2a-c). Significant charging demand near the Beijing Capital International Airport has not currently been covered by the existing stations (Figure 2d).

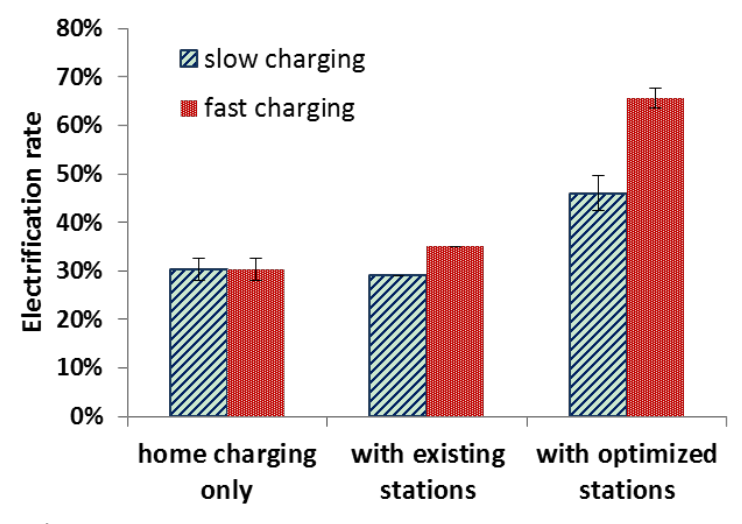

a)

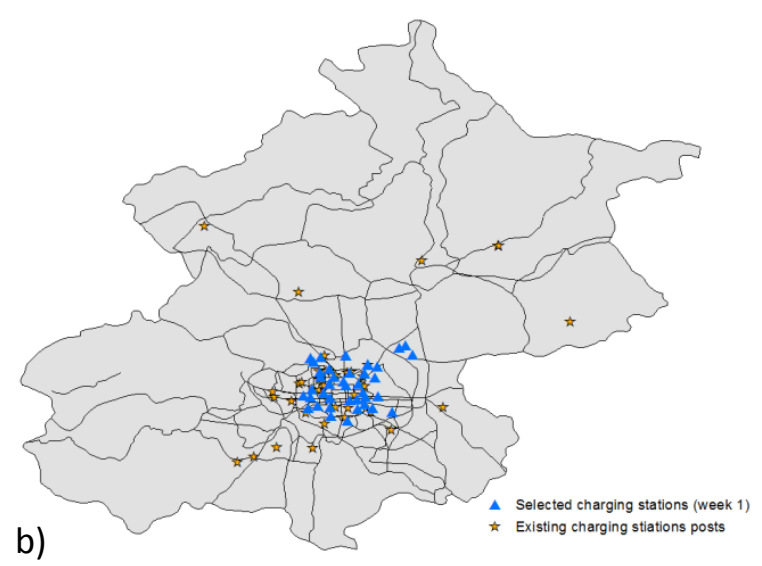

b)

Figure 1. Electrified fleet VMT as percentage of total fleet VMT (electrification rate) (a) and optimal public charging stations (b) of the 40-station scenario comparing to the existing 40 built charging stations. 

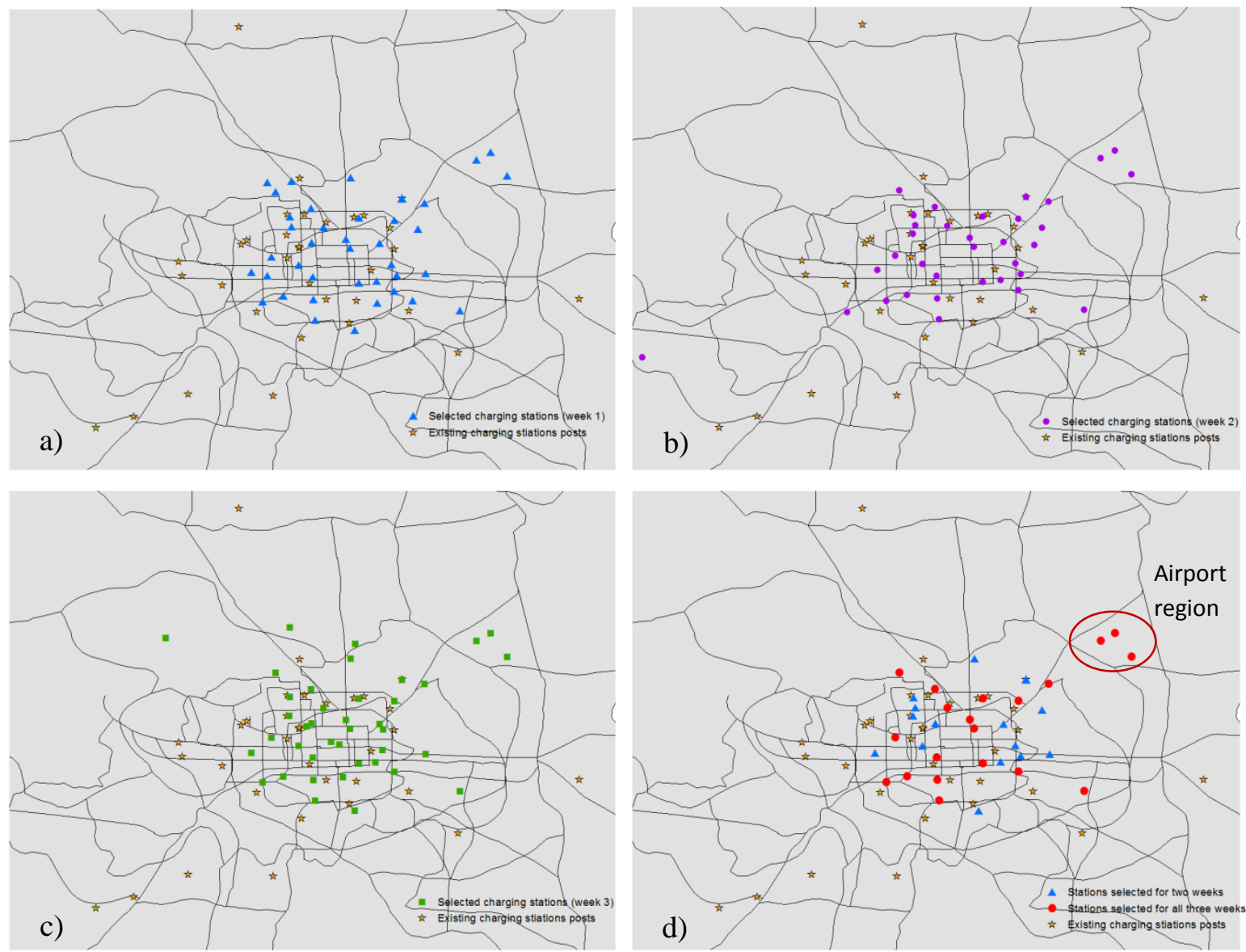

Figure 2. Location comparison of optimized stations and existing stations in the inner city of Beijing: a) optimization results with data for week 1 ; b) optimization results with data for week 2; c) optimization results with data for week; and d) optimal locations selected in two or three weeks.

\subsection{Impacts of increased number of charging stations}

To evaluate the impact of the total number of stations that can be installed $(B)$ on the optimization results, we ran models ranging $B$ from 20 to 500 . The results show that, while increasing the total number of charging stations increases electrified fleet VMT regardless of charging speed, the marginal electrified fleet VMT for both type of charging stations quickly diminishes (Figure 3). The difference in electrified fleet VMT between installing the same number of fast and slow charging stations diverges initially but stays stable at about $20 \%$ when there are 40 or more charging stations. 


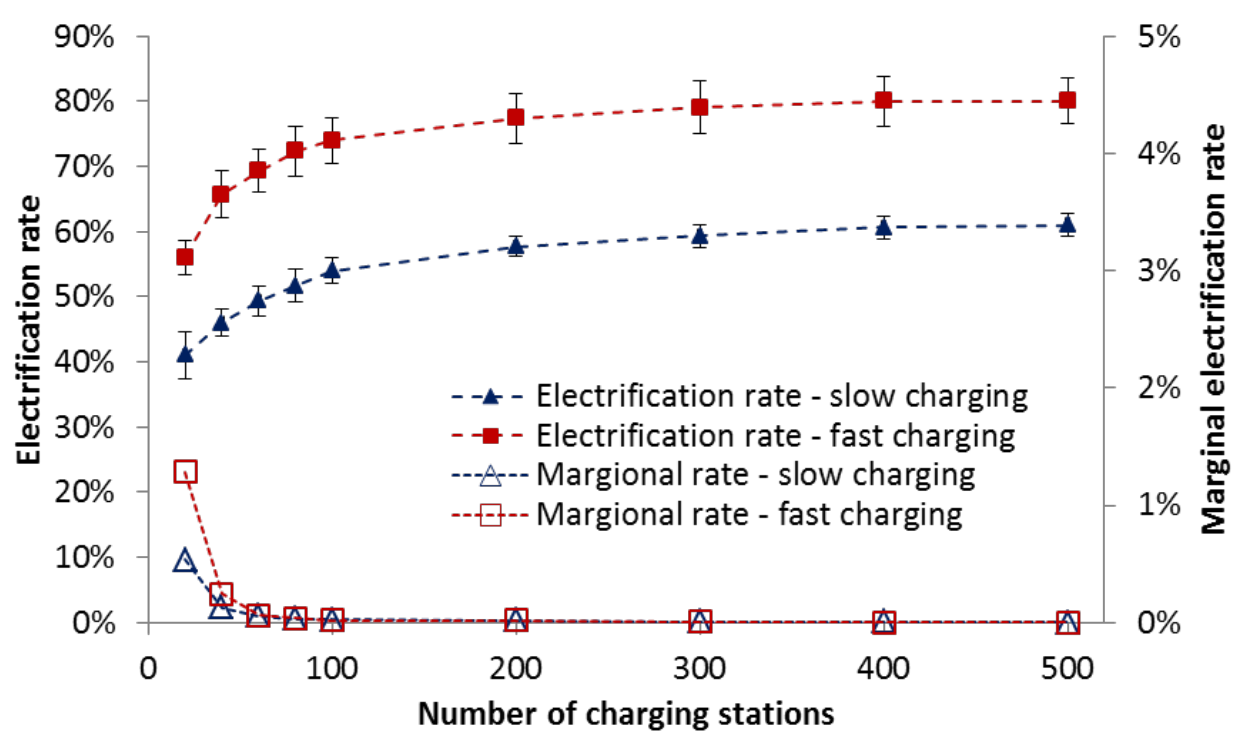

Figure 3. Change of electrified fleet VMT as the percentage of total fleet VMT (electrification rate) and marginal electrification rate with increasing number of charging stations

The locations of optimized stations concentrate in the inner city in all scenarios, gradually expanding to the suburban area with increasing number of charging stations (Figure 4a-4d). Zoomed-in maps for the inner city are included in the Supplemental Information (Figure SI2). To measure how many of the selected stations stay as the optimal choice when the total number of charging stations is increased, we define retention rate as the percentage of selected stations in a scenario with smaller number of stations remains as the optimal choices in a scenario with greater number of stations. As shown in Figure 5, on average, the overall retention rate is $70 \%$ to $88 \%$ for slow charging and $67 \%$ to $88 \%$ for fast charging, which indicates that the majority of the optimal stations are consistently selected even when the total number of charging stations are increased by 25 times. In general, the optimal slow charging stations have higher retention rate than the fast charging ones, showing that slow charging stations selected for short term planning (i.e. scenarios with less total number of stations) are more likely to stay as the optimal choices for long term planning (i.e. scenarios with larger total number of stations). Additionally, the standard deviation of the retention rate reduces with increasing total number of charging stations, showing that the variation of travel pattern among different weeks can be better covered with more charging stations. With 200 charging stations, the standard deviation of retention rate can be effectively reduced to less than $3 \%$. 


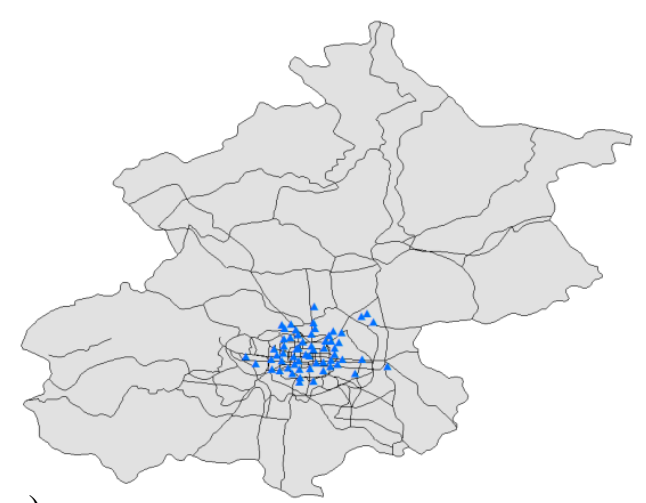

a)

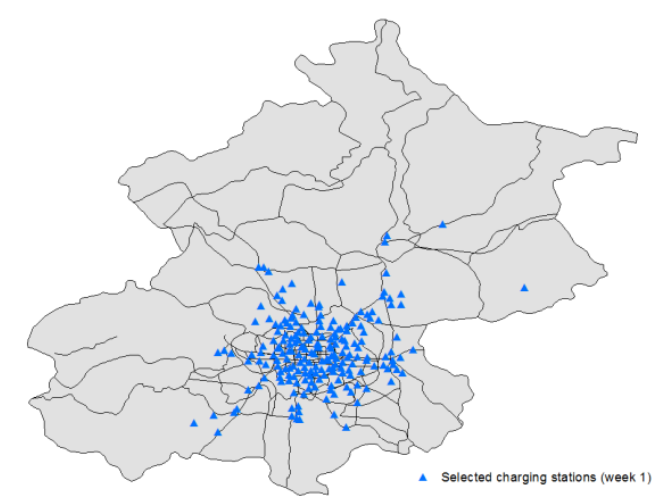

c)

Figure 4. Locations of optimized stations in scenarios with different total number of charging stations: a) 60 stations, b) 100 stations, c) 200 stations, and d) 500 stations.
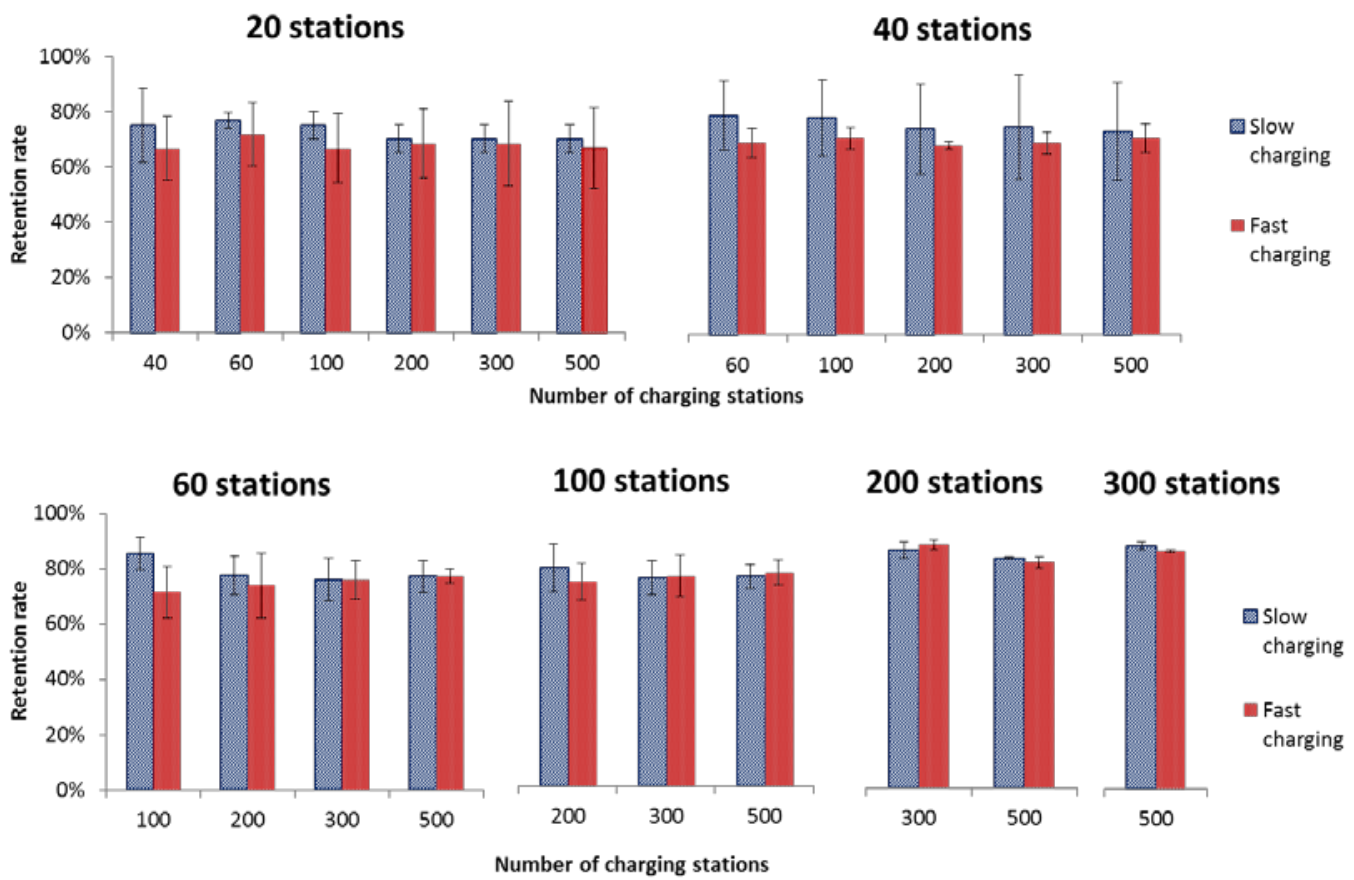

Figure 5. Retention rate of selected stations when increasing total number of charging stations 


\subsection{Impacts of AER}

AER is also a key factor in determining overall electrification rate. To test the model's sensitivity to AER, we conducted a sensitivity analysis ranging AER from 60 miles to 120 miles. The results show that, in the tested range, greater AER can lead to higher electrification rate (Figure 6). On average, increasing AER by 1 mile can gain $0.15 \%$ to $0.4 \%$ of electrification rate improvement with fast charging, and $0.15 \%$ to $0.25 \%$ with slow charging. It is notable that PHEVs with greater AER could be more expensive and as a result have lower adoption rates (Cai and $\mathrm{Xu}, 2013$ ). The economics consideration is not included in this analysis. With the same number of charging stations, the locations of the selected stations are not significantly affected by AER (Figure 7). With fast charging, 63\% to $80 \%$ of the selected stations are consistent regardless of different AER; while with slow charging, the rate is higher at $75 \%$ to $82 \%$.
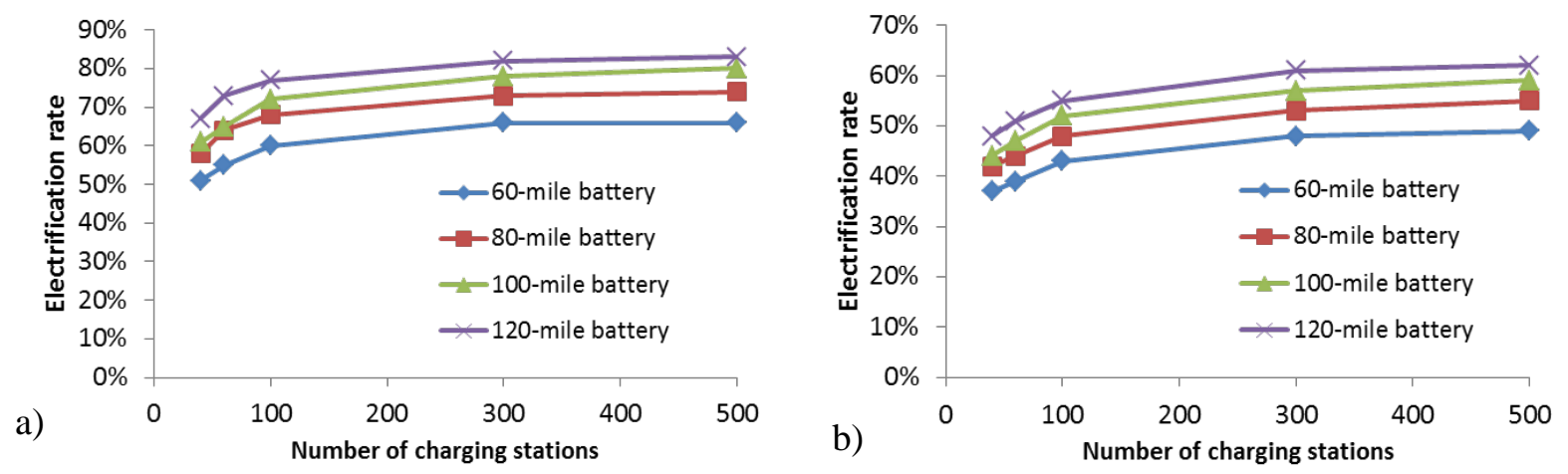

Figure 6. Electrification rates with different battery all-electric-range and different numbers of charging stations: a) fast charging; b) slow charging.
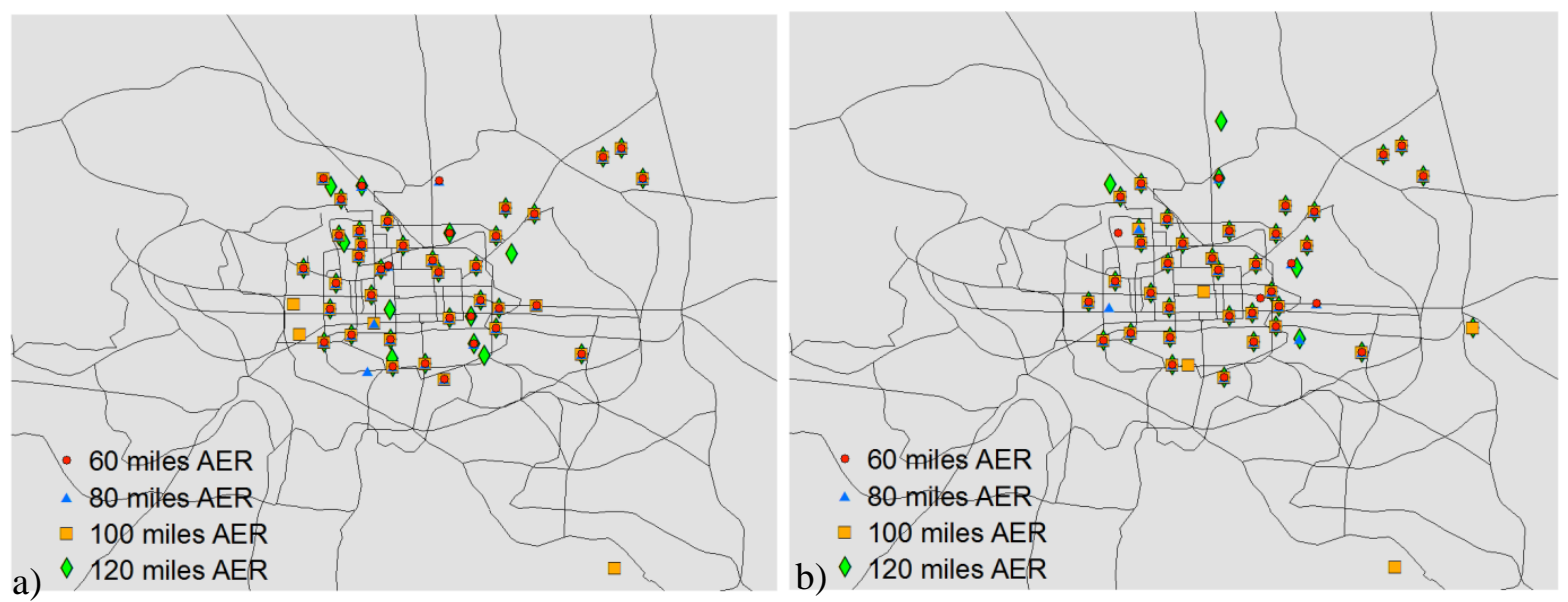

Figure 7. Locations of selected stations in 40-station scenario using different AER: a) fast charging; b) slow charging.

\subsection{Limitations and future directions}

While the proposed model has the merit of incorporating real world vehicle travel behavior to better estimate public charging demand, a key assumption is that the drivers will not change their behavior when they switch from conventional gasoline vehicles to PHEVs. When drivers 
do not need to change their travel patterns to accommodate the use of EVs, they are more likely to adopt. However, it is possible that PHEV drivers may modify their behavior to actively seek charging opportunities, because electricity is cheaper than gasoline on a per mile basis. Future studies to compare travel behavior before and after EV adoption will be needed to evaluate the impact of this assumption.

The case study of taxi fleet in Beijing, China demonstrates that real world vehicle trajectories can be used in optimization models to estimate charging demand. However, the results of the case study need to be used carefully for policy recommendations. First of all, the case study assumes that the public charging infrastructure only serves the taxi fleet. The charging demand of other vehicles (e.g. private vehicles, public service vehicles, buses) is not considered. Future research incorporating data of different fleets will be needed to better reflect city level public charging infrastructure for mixed uses. In addition, this model does not take into consideration the space constraints and the capacity limits of the charging stations. Depending on the location of the existing gas stations, certain stations may be constrained by space, the number of charging posts, and the associated parking space it can accommodate. If all charging posts are occupied at a given time, nearby vehicles with charging needs will not be able to charge at this station. Furthermore, the candidate locations in this case study are limited to existing gas stations only. Additional candidates can be explored. For example, when modeling private vehicles, existing parking lots may be better candidates for charging stations.

Additionally, the representativeness of the data needs to be future verified. In total, Beijing has around 66,000 taxis. While the data used in this study contains $18 \%$ of the fleet and no specific biases are observed, the representativeness of sampled taxis need to be further examined with additional datasets.

Lastly, the maximized electrified fleet VMT does not guarantee maximized environmental benefits. Higher electrified fleet VMT displaces more fossil-based liquid fuels (e.g., gasoline). However, the environmental impacts of fleet electrification depend on the grid fuel mix, charging time (base load versus peak load), and individual driving conditions (variations of fuel economy). Future studies can expand the model to optimize environmental benefits.

\section{Conclusion}

In this study, an optimization model is formulated to find the optimal locations for installing charging stations to maximize electrified fleet VMT based on vehicle travel behaviors. The formulated model is implemented for Beijing, China as a case study using vehicle trajectory data of 11,880 taxis over a period of three weeks. The large scale data is handled by formulating the mathematical model properly, transforming input data, and adjusting Cplex options. The results show that the optimal locations of charging stations can have significant improvements. The Majority of the optimal locations are selected in the inner city. By increasing the total number of charging stations, the locations of the optimal stations expand outward from the inner city. While more charging stations increase electrified fleet VMT, the marginal gain diminishes quickly regardless of charging speed. With more than 40 charging stations, the difference of electrified fleet VMT between the same number of slow and fast 
charging stations stays constantly at $20 \%$. The majority of the stations selected in a model with smaller number of charging stations remain as the optimal choices when the total number of charging stations increases. Slow charging stations generally have higher retention rates.

\section{Acknowledgment}

This material is based upon work partially supported by the Department of Energy under Award Number DE-PI0000012. NS and MT acknowledge the financial support for this work from the European Commission LOG4GREEN Project Grant \#287091 under FP7 program. HC thanks the support of the Dow Sustainability Fellows Program.

\section{References}

A Krieger, L Wang, P Radtke, C.M., 2012. Recharging China’s electric vehicle aspirations: A perspective on revitalizing China's electric vehicle industry. McKinsey Co. China Auto Hub.

Cai, H., Jia, X., Chiu, A.S.F., Hu, X., Xu, M., 2014. Siting public electric vehicle charging stations in Beijing using big-data informed travel patterns of the taxi fleet. Transp. Res. Part D Transp. Environ. 33, 39-46. doi:10.1016/j.trd.2014.09.003

Cai, H., Xu, M., 2013. Greenhouse gas implications of fleet electrification based on big datainformed individual travel patterns. Environ. Sci. Technol. 47, 9035-43. doi:10.1021/es401008f

City of Surrey, 2012. Electric Vehicles [WWW Document]. URL http://www.surrey.ca/community/12250.aspx (accessed 8.27.14).

Deutsche Bank Group, 2012. China’s Green Move - Vehicle Electrification Ahead.

Dong, J., Liu, C., Lin, Z., 2014. Charging infrastructure planning for promoting battery electric vehicles: An activity-based approach using multiday travel data. Transp. Res. Part C Emerg. Technol. 38, 44-55. doi:10.1016/j.trc.2013.11.001

He, F., Wu, D., Yin, Y., Guan, Y., 2013. Optimal deployment of public charging stations for plug-in hybrid electric vehicles. Transp. Res. Part B Methodol. 47, 87-101. doi:10.1016/j.trb.2012.09.007

Hoogma, R., Kemp, R., Schot, J., Truffer, B., 2005. Experimenting for Sustainable Transport: The Approach of Strategic Niche Management. Routledge.

JR, E.G.B., 2012. \$120 Million Settlement to Fund Electric Car Charging Stations Across California [WWW Document]. URL http://gov.ca.gov/news.php?id=17463 (accessed 8.27.14). 
Kelly, J.C., MacDonald, J.S., Keoleian, G.A., 2012. Time-dependent plug-in hybrid electric vehicle charging based on national driving patterns and demographics. Appl. Energy 94, 395-405. doi:10.1016/j.apenergy.2012.02.001

Markel, T., 2010. Plug-in Electric Vehicle Infrastructure : A Foundation for Electrified Transportation Preprint.

Morrow, K., 2008. U . S . Department of Energy Vehicle Technologies Program - Advanced Vehicle Testing Activity Plug-in Hybrid Electric Vehicle Charging Infrastructure Review.

NAATBatt International, 2015. Advanced Battery Weekly.

Navigant Research Group, 2013. More than 1.8 Million Plug-In Electric Vehicles Will Be Sold in the Largest 102 U.S. Cities From 2012 to 2020 Navigant Research.

Sadeghi-Barzani, P., Rajabi-Ghahnavieh, A., Kazemi-Karegar, H., 2014. Optimal fast charging station placing and sizing. Appl. Energy 125, 289-299.

doi:10.1016/j.apenergy.2014.03.077

Solutions, K.M., Remote, C., Server, O., 2012. Cplex 12.

State Grid Corporation of China, 2010. Technical specification for electric vehicle charging spot (in Chinese), 2010th ed.

Tong, S., 2014. Beijing: New Energy Vehicles Have Seperate Quota Starting in Feburary 2014 [WWW Document]. URL http://news.xinhuanet.com/politics/201401/29/c_126076733.htm

Torchio, M.F., Santarelli, M.G., 2010. Energy, environmental and economic comparison of different powertrain/fuel options using well-to-wheels assessment, energy and external costs - European market analysis. Energy 35, 4156-4171.

doi:10.1016/j.energy.2010.06.037

USA.gov, 2013. New Plug-in Hybrids [WWW Document]. URL http://www.fueleconomy.gov/feg/phevtech.shtml (accessed 8.28.14).

Wang, H., Er, S.T.M., Huang, Q.I., Er, S.E.M., Zhang, C., Xia, A., 2010. A NOVEL APPROACH FOR THE LAYOUT OF ELECTRIC VEHICLE CHARGING STATION $64-70$.

WantChinaTimes, 2013. 200,000 electric cars on Beijing's roads by 2017 [WWW Document]. URL http://www.wantchinatimes.com/news-subclasscnt.aspx?id=20131114000055\&cid=1105 (accessed 6.3.15).

Xi, X., Sioshansi, R., Marano, V., 2013. Simulation-optimization model for location of a public electric vehicle charging infrastructure. Transp. Res. Part D Transp. Environ. 22, 60-69. doi:10.1016/j.trd.2013.02.014 